\title{
Entropy production and asymptotic factorization via thermalization: A collisional model approach
}

\author{
Stefano Cusumano, ${ }^{1, *}$ Vasco Cavina, ${ }^{1}$ Maximilian Keck, ${ }^{1}$ Antonella De Pasquale, ${ }^{2,3,1}$ and Vittorio Giovannetti ${ }^{1}$ \\ ${ }^{1}$ NEST, Scuola Normale Superiore and Istituto Nanoscienze-CNR, I-56127 Pisa, Italy \\ ${ }^{2}$ Dipartimento di Fisica e Astronomia, Universitá di Firenze, I-50019 Sesto Fiorentino (FI), Italy \\ ${ }^{3}$ INFN Sezione di Firenze, via G.Sansone 1, I-50019 Sesto Fiorentino (FI), Italy
}

(Received 12 July 2018; published 26 September 2018)

\begin{abstract}
The Markovian evolution of an open quantum system is characterized by a positive entropy production, while the global entropy gets redistributed between the system and the environment degrees of freedom. Starting from these premises, we analyze the entropy variation of an open quantum system in terms of two distinct relations: the Clausius inequality, that provides an intrinsic bound for the entropy variation in terms of the heat absorbed by the system, and an extrinsic inequality, which instead relates the former to the corresponding entropy increment of the environment. By modeling the thermalization process with a Markovian collisional model, we compare and discuss the two bounds, showing that the latter is asymptotically saturated in the limit of large interaction time. In this regime not only the reduced density matrix of the system reaches an equilibrium configuration, but it also factorizes from the environment degrees of freedom. This last result is proven analytically when the system-bath coupling is sufficiently strong and through numerical analysis in the weak-coupling regime.
\end{abstract}

DOI: 10.1103/PhysRevA.98.032119

\section{INTRODUCTION}

In recent times research in thermodynamics has risen to new life, with special attention to the energy balance in nanoscale devices and a growing interest towards its connections to information theory [1]. In this framework an issue naturally arises about the relations between thermodynamic and information theoretic quantities, for instance, between the concept of entropy introduced by Clausius and the one introduced by Shannon [2,3]. Such studies are complicated by the fact that for open quantum dynamical processes [4] a definition of heat and work for a quantum engine cannot be unequivocally given in the presence of correlations and non-negligible coupling strengths [5-11], or more in general when the system is driven out of equilibrium [12].

In this work we focus our attention on the irreversible character of the thermalization process, by studying how the entropy of a quantum system A is modified when it is placed into thermal contact with an external reservoir. Specifically, the entropy increase of A is analyzed in terms of two different inequalities: the Clausius formulation of the second law and one that accounts for creation of quantum and classical correlations between A and its environment. A detailed analysis of these relations is performed for the thermalization scheme via collisional events introduced by Scarani $e t$ al. in Ref. [13]. Collisional models (CMs) [14-16] have been extensively used to study open quantum systems in a variety of situations, from cascade systems and networks [17-19], to heat transfer [20] and thermalization [13,21]. Apart from their intrinsic simplicity, they allow for exact tracking of the environmental degrees of freedom, a fundamental feature that lets one account for

*Corresponding author: stefano.cusumano@sns.it the entropy and energy balance in the system. Via exact analytic results extended by numerical analysis we get an insight into the final state of the system and the bath after the thermalization process is completed: in particular we observe that for the scheme of Ref. [13] the final state of A not only reaches thermalization locally, but also gets completely factorized from the many-body environment that triggers the thermalization via collisions (an effect we dub asymptotic factorization of $\mathrm{A}$ ). An explicit proof of this phenomenon is given which works in the strong-collisions limit regime. We conjecture that the same result should apply also in the weak-collisions limit and present numerical evidence of this fact for the special case where A is a qubit system.

The manuscript is organized as follows. In Sec. II, using a standard Hamiltonian characterization of the thermalization process, we present two different bounds on the entropy increase of A and clarify their relations. In Sec. III instead we review the CM of Ref. [13] and study how the previous bounds affect the dynamics of the system in this setting. Section IV is devoted to the study of the asymptotic factorization property. The paper ends in Sec. V, where we draw our conclusions. Technical derivations are reported in the Appendix.

\section{QUANTIFYING IRREVERSIBILITY}

Consider a quantum system A weakly coupled to a thermal environment B at temperature $T$. Suppose that, due to its interaction with $\mathrm{B}$, the system evolves with an entropy increase $\Delta S_{\mathrm{A}}$ and absorbing an amount of heat $\Delta Q_{\mathrm{A}}$. Purely thermodynamic considerations suggest that

$$
\Delta S_{\mathrm{A}} \geqslant \beta \Delta Q_{\mathrm{A}} \quad \text { (Clausius inequality) }
$$

as a consequence of the second law [hereafter we use $\beta=$ $1 /\left(K_{B} T\right)$ to indicate the inverse temperature of the bath, 
$K_{B}$ being the Boltzmann constant]. Equation (1) provides an "intrinsic" lower bound on the local entropy production as it involves only quantities that explicitly refer to properties of the system A. By properly accounting for the onset of classical and quantum correlations during the thermalization event, an "extrinsic" bound relating $\Delta S_{\mathrm{A}}$ to the corresponding entropy increase of the bath $\Delta S_{\mathrm{B}}$ can also be obtained, leading to the inequality

$$
\Delta S_{\mathrm{A}} \geqslant-\Delta S_{\mathrm{B}}
$$

(more on this in the following). Limiting how the entropy of A evolves, Eqs. (1) and (2) provide a characterization of the irreversibility of the thermalization process. As we shall clarify later on they are not completely independent, even though no universal ordering between them can be established. A formal derivation of these results can be obtained by modeling the $\mathrm{AB}$ coupling as a time-independent Hamiltonian $\hat{H}=\hat{H}_{\mathrm{A}}+\hat{H}_{\mathrm{B}}+\hat{H}_{\text {int }}$ and assuming that no correlations are shared between $\mathrm{A}$ and $\mathrm{B}$ before their interaction, i.e., writing the initial state of the joint system as a factorized density matrix

$$
\hat{\rho}_{\mathrm{AB}}(0)=\hat{\rho}_{\mathrm{A}}(0) \otimes \hat{\eta}_{\mathrm{B}}^{(\beta)},
$$

where $\hat{\rho}_{\mathrm{A}}(0)$ is the input state of $\mathrm{A}$, while $\hat{\eta}_{\mathrm{B}}^{(\beta)}:=$ $e^{-\beta \hat{H}_{\mathrm{B}}} / Z_{\mathrm{B}}(\beta)$ is the Gibbs density matrix describing the thermal equilibrium of the bath, with $Z_{\mathrm{B}}(\beta):=\operatorname{Tr}\left[e^{-\beta \hat{H}_{\mathrm{B}}}\right]$ being the partition function. With this specification the temporal evolution of A can now be fully specified by the oneparameter family of completely positive, trace (CPT) preserving channels $\left\{\Phi_{0 \rightarrow t}\right\}_{t \geqslant 0}$ describing, for arbitrary times $t$, the mapping

$$
\hat{\rho}_{\mathrm{A}}(0) \rightarrow \hat{\rho}_{\mathrm{A}}(t)=\Phi_{0 \rightarrow t}\left[\hat{\rho}_{\mathrm{A}}(0)\right]:=\operatorname{Tr}_{B}\left[\hat{\rho}_{\mathrm{AB}}(t)\right],
$$

with $\hat{\rho}_{\mathrm{AB}}(t):=e^{-i \hat{H} t / \hbar}\left(\hat{\rho}_{\mathrm{A}}(0) \otimes \hat{\eta}_{\mathrm{B}}^{(\beta)}\right) e^{i \hat{H} t / \hbar} \quad$ being the evolved state of the joint system. We then say that $\mathrm{B}$ induces thermalization on $\mathrm{A}$ if, irrespective from the specific choice of $\hat{\rho}_{\mathrm{A}}(0)$, the latter will be driven by $\Phi_{0 \rightarrow t}$ into the equilibrium configuration state $\hat{\eta}_{\mathrm{A}}^{(\beta)}:=e^{-\beta \hat{H}_{\mathrm{A}}} / Z_{\mathrm{A}}(\beta)$, possibly in the asymptotic limit of an infinitely long interaction time $t \rightarrow \infty$. Under this premise, since no external work contributes to the energy balance, the heat absorbed by A can be legitimately identified with the increases of the local energy of A, i.e. $[6,22]$,

$$
\Delta Q_{\mathrm{A}}=\operatorname{Tr}\left[\hat{H}_{\mathrm{A}}\left(\hat{\rho}_{\mathrm{A}}(t)-\hat{\rho}_{\mathrm{A}}(0)\right)\right] .
$$

The conservation of the total energy in the model, i.e., $\operatorname{Tr}\left[\hat{H}\left(\hat{\rho}_{\mathrm{AB}}(t)-\hat{\rho}_{\mathrm{AB}}(0)\right)\right]=0$, implies that

$$
\Delta Q_{\mathrm{A}}=-\Delta Q_{\mathrm{B}}-\Delta E_{\mathrm{int}},
$$

where $\Delta Q_{\mathrm{B}}:=\operatorname{Tr}\left[\hat{H}_{\mathrm{B}}\left(\hat{\rho}_{\mathrm{B}}(t)-\hat{\rho}_{\mathrm{B}}(0)\right)\right]$ is the heat absorbed by the bath in the process, while the energy contribution

$$
\Delta E_{\mathrm{int}}:=\operatorname{Tr}\left[\hat{H}_{\mathrm{int}}\left(\hat{\rho}_{\mathrm{AB}}(t)-\hat{\rho}_{\mathrm{AB}}(0)\right)\right]
$$

holds a less clear operational interpretation. In the weakcoupling regime, the latter term is typically neglected either because it is assumed to be small as compared to $\Delta Q_{\mathrm{A}, \mathrm{B}}$ or, more formally, by simply considering interaction Hamiltonians that only exchange excitations between $\mathrm{A}$ and $\mathrm{B}$ (i.e., $\left.\left[\hat{H}_{\mathrm{int}}, \hat{H}_{\mathrm{A}}+\hat{H}_{\mathrm{B}}\right]=0\right)$, leading to the identity $\Delta Q_{\mathrm{A}}=$
$-\Delta Q_{\mathrm{B}}$. The Clausius inequality can then be derived by interpreting the left-hand side (LHS) term of (1) as the variation of the von Neumann entropy computed on the initial state $\hat{\rho}_{\mathrm{A}}(0)$ and its evolved counterpart $\hat{\rho}_{\mathrm{A}}(t)$, i.e., $\Delta S_{A}=S\left(\hat{\rho}_{\mathrm{A}}(t)\right)-$ $S\left(\hat{\rho}_{\mathrm{A}}(0)\right)$ with $S\left(\hat{\rho}_{\mathrm{A}}(t)\right):=-\operatorname{Tr}\left[\hat{\rho}_{\mathrm{A}}(t) \ln \hat{\rho}_{\mathrm{A}}(t)\right]$. Accordingly we can now write

$$
\Delta S_{\mathrm{A}}-\beta \Delta Q_{\mathrm{A}}=S\left(\hat{\rho}_{\mathrm{A}}(0) \| \hat{\eta}_{\mathrm{A}}^{(\beta)}\right)-S\left(\hat{\rho}_{\mathrm{A}}(t) \| \hat{\eta}_{\mathrm{A}}^{(\beta)}\right),
$$

where $S\left(\hat{\rho}_{\mathrm{A}} \| \hat{\eta}_{\mathrm{A}}^{(\beta)}\right):=\operatorname{Tr}\left[\hat{\rho}_{\mathrm{A}}\left(\ln \hat{\rho}_{\mathrm{A}}-\ln \hat{\eta}_{\mathrm{A}}^{(\beta)}\right)\right]$ is the relative entropy [23] of the density matrices $\hat{\rho}_{\mathrm{A}}$ and $\hat{\eta}_{\mathrm{A}}^{(\beta)}$. Assuming the invariance of $\hat{\eta}_{\mathrm{A}}^{(\beta)}$ under $\Phi_{0 \rightarrow t}$ (which given our working hypothesis should hold at least in the asymptotic limit of $t \rightarrow \infty$ ), the inequality (1) then follows as a consequence of the monotonicity property of the relative entropy under CPT transformations [4], i.e.,

$$
\begin{aligned}
S\left(\hat{\rho}_{\mathrm{A}}(t) \| \hat{\eta}_{\mathrm{A}}^{(\beta)}\right) & =S\left(\Phi_{0 \rightarrow t}\left[\hat{\rho}_{\mathrm{A}}(0)\right] \| \Phi_{0 \rightarrow t}\left[\hat{\eta}_{\mathrm{A}}^{(\beta)}\right]\right) \\
& \leqslant S\left(\hat{\rho}_{\mathrm{A}}(0) \| \hat{\eta}_{\mathrm{A}}^{(\beta)}\right) .
\end{aligned}
$$

Equation (8) also clarifies that, at least in the asymptotic limit where $\hat{\rho}_{\mathrm{A}}(t)$ reaches thermalization, the Clausius bound will in general not be tight, as in this case Eq. (8) reduces to

$$
\Delta S_{\mathrm{A}}-\left.\beta \Delta Q_{\mathrm{A}}\right|_{t \rightarrow \infty}=S\left(\hat{\rho}_{\mathrm{A}}(0) \| \hat{\eta}_{\mathrm{A}}^{(\beta)}\right),
$$

which due to the positivity of the relative entropy is not null, unless $\hat{\rho}_{\mathrm{A}}(0)$ was already at equilibrium. On the other hand, the extrinsic bound (2) admits an even simpler derivation: it follows as a consequence of the subadditivity property of the von Neumann entropy and its invariance under unitary transformations [23], i.e., $S\left(\hat{\rho}_{\mathrm{A}}(t)\right)+S\left(\hat{\rho}_{\mathrm{B}}(t)\right) \geqslant$ $S\left(\hat{\rho}_{\mathrm{AB}}(t)\right)=S\left(\hat{\rho}_{\mathrm{AB}}(0)\right)=S\left(\hat{\rho}_{\mathrm{A}}(0)\right)+S\left(\hat{\rho}_{\mathrm{B}}(0)\right)$ or, equivalently, from the positivity of the quantum mutual information [23]

$$
0 \leqslant I_{\mathrm{A}: \mathrm{B}}(t):=S\left(\hat{\rho}_{\mathrm{A}}(t)\right)+S\left(\hat{\rho}_{\mathrm{B}}(t)\right)-S\left(\hat{\rho}_{\mathrm{AB}}(t)\right),
$$

with $\hat{\rho}_{\mathrm{B}}(t)=\operatorname{Tr}_{\mathrm{A}}\left[\hat{\rho}_{\mathrm{AB}}(t)\right]$ the reduced density matrix of the bath at time $t$. As anticipated (1) and (2) are not independent: following the derivation of Ref. [24] it is possible to show that the difference between their right-hand-side terms satisfies the identity

$$
\beta \Delta Q_{\mathrm{A}}+\Delta S_{\mathrm{B}}=-S\left(\hat{\rho}_{\mathrm{B}}(t) \| \hat{\eta}_{\mathrm{B}}^{(\beta)}\right)-\beta \Delta E_{\mathrm{int}}
$$

[indeed by invoking the energy conservation identity $\Delta Q_{\mathrm{B}}=$ $-\Delta Q_{\mathrm{A}}-\Delta E_{\mathrm{int}}$ and exploiting the connection between $I_{\mathrm{A}: \mathrm{B}}(t), \Delta S_{\mathrm{A}}$, and $\Delta S_{\mathrm{B}}$ detailed in Eq. (11), the identity (12) reduces to the expression $\beta \Delta Q_{\mathrm{B}}=-\Delta S_{\mathrm{A}}+$ $I_{\mathrm{A}: \mathrm{B}}(t)+S\left(\hat{\rho}_{\mathrm{B}}(t) \| \hat{\eta}_{\mathrm{B}}^{(\beta)}\right)$ of [24]]. Notice that, for general choices of the system-bath coupling, the term on the RHS of Eq. (12) does not have a definite sign with the notable exceptions of those models for which $\Delta E_{\text {int }}$ is exactly zero (e.g., the case where $\hat{H}_{\text {int }}$ commutes with $\hat{H}_{\mathrm{A}}$ and $\hat{H}_{\mathrm{B}}$ ): under this circumstance the positivity of the relative entropy ensures that $\beta \Delta Q_{\mathrm{A}}$ is smaller than $-\Delta S_{\mathrm{B}}$ making the extrinsic bound (2) tighter than (1).

\section{Markovian regime}

Let us focus on the case of a thermalizing process described by CPT families of maps $\left\{\Phi_{0 \rightarrow t}\right\}$ which are time homogeneous and Markovian [25]. As they keep no record of the 
initial time (i.e., $\Phi_{0 \rightarrow t}=\Phi_{t}$ ) and obey a semigroup property (i.e., $\Phi_{t+\tau}=\Phi_{t} \circ \Phi_{\tau}$ for all $t, \tau \geqslant 0$, with "o" indicating the composition of superoperator), the corresponding dynamics can be expressed in terms of a master equation [4] with a Gorini-Kossakowsky-Sudarshan-Lindblad (GKSL) generator $[26,27]$ formed by a local Hamiltonian contribution $\hat{H}_{\mathrm{A}}$ and a purely dissipative term which effectively accounts for the influence of the bath $\mathrm{B}$. Under these assumptions the derivation of the Clausius inequality given in the previous section can be generalized to bound the differential entropy increase $\partial S_{\mathrm{A}}(t):=S\left(\hat{\rho}_{\mathrm{A}}(t+d t)\right)-S\left(\hat{\rho}_{\mathrm{A}}(t)\right)$ at a generic time $t \geqslant 0$ of the temporal evolution of $\mathrm{A}$ in terms of the corresponding differential heat increment $\partial Q_{\mathrm{A}}(t):=$ $\operatorname{Tr}\left[\hat{H}_{\mathrm{A}}\left(\hat{\rho}_{\mathrm{A}}(t+d t)-\hat{\rho}_{\mathrm{A}}(t)\right)\right]$, i.e.,

$$
\partial S_{\mathrm{A}}(t) \geqslant \beta \partial Q_{\mathrm{A}}(t) .
$$

This supersedes the finite time interval version of the bound which can be now derived from (13) by direct integration, and it implies that, for time-homogeneous Markovian processes, the gap between the RHS and the LHS of the Clausius inequality is a nondecreasing function of time. An analogous treatment of Eq. (2) is more problematic as the Hamiltonian derivation of GKSL master equation relies on special assumptions on the $\mathrm{AB}$ couplings that hide the modifications induced on the bath degrees of freedom. For properly accounting these effects we need a framework that permits one to treat all the degrees of freedom, including the bath ones, on an equal footing, e.g., exploiting the CM approach we analyze in the following section.

\section{THERMALIZATION VIA COLLISIONS}

CMs are simplified yet effective descriptions of the $\mathrm{AB}$ interactions $[14,28-30]$. Here the thermal bath $B$ is depicted as a many-body quantum system formed by a huge collection of $N$ (possibly infinite) identical and noninteracting, small subsystems $b_{1}, b_{2}, \ldots, b_{N}$, characterized by local Hamiltonians $\hat{H}_{\mathrm{b}_{1}}, \hat{H}_{\mathrm{b}_{2}}, \ldots, \hat{H}_{\mathrm{b}_{N}}$ and initialized in the same Gibbs thermal state $\hat{\eta}_{\mathrm{b}}^{(\beta)}:=e^{-\beta \hat{H}_{\mathrm{b}}} / Z_{\mathrm{b}}(\beta)$. Such subsystems interact sequentially with $\mathrm{A}$ for a finite time $\delta t$ and are then discarded to enforce Markovianity $[4,13,21,31]$. Within this model the global state of the joint system $\mathrm{AB}$ after the first $n$ collisions is hence expressed as

$$
\hat{\rho}_{\mathrm{AB}}^{(n)}=\mathcal{U}_{n} \circ \cdots \circ \mathcal{U}_{2} \circ \mathcal{U}_{1}\left[\hat{\rho}_{\mathrm{A}}^{(0)} \otimes\left(\hat{\eta}_{\mathrm{b}}^{(\beta)}\right)^{\otimes N}\right],
$$

with $\hat{\rho}_{A}^{(0)}$ and $\left(\hat{\eta}_{\mathrm{b}}^{(\beta)}\right)^{\otimes N}$ being respectively the initial density matrices of $\mathrm{A}$ and $\mathrm{B}$, and where, for $k$ an integer, $\mathcal{U}_{k}(\cdots)=$ $\hat{U}_{k}(\cdots) \hat{U}_{k}^{\dagger}$ is a unitary conjugation induced by the interaction between $\mathrm{A}$ and the $k$ th bath subsystem $\mathrm{b}_{k}$. Accordingly A will now evolve via a stroboscopic sequence of jumps in which, at the $n$th step, the reduced density matrix $\hat{\rho}_{\mathrm{A}}^{(n-1)}:=\operatorname{Tr}_{\mathrm{B}}\left[\hat{\rho}_{\mathrm{AB}}^{(n-1)}\right]$ gets mapped into

$$
\hat{\rho}_{\mathrm{A}}^{(n)}=\operatorname{Tr}_{\mathrm{b}}\left[\mathcal{U}_{n}\left(\hat{\rho}_{\mathrm{A}}^{(n-1)} \otimes \hat{\eta}_{\mathrm{b}}^{(\beta)}\right)\right]=: \Phi\left[\hat{\rho}_{\mathrm{A}}^{(n-1)}\right],
$$

where we decomposed the partial trace upon B into a sequence of partial traces upon the various bath subsystems to remove all the degrees of freedom but the $n$th ones. Equation (15) makes explicit the Markovian character of the evolution and, by iteration, clarifies that in the $\mathrm{CM}$ the elements of the family $\left\{\Phi_{t}\right\}_{t \geqslant 0}$ are replaced by the powers of the map $\Phi$, i.e., $\hat{\rho}_{\mathrm{A}}^{(n)}=$ $\Phi^{n}\left[\hat{\rho}_{\mathrm{A}}^{(0)}\right]$.

In order to ensure that for large enough $n$ the system A will reach the thermal equilibrium state $\hat{\eta}_{\mathrm{A}}^{(\beta)}$, i.e.,

$$
\lim _{n \rightarrow \infty} \hat{\rho}_{\mathrm{A}}^{(n)}=\hat{\eta}_{\mathrm{A}}^{(\beta)},
$$

we follow Refs. [13,31-35] assuming the environment subsystems $b_{k}$ to be isomorphic with $\mathrm{A}$, identifying their local Hamiltonians with the one of A (i.e., $\hat{H}_{\mathrm{A}} \equiv \hat{H}_{\mathrm{b}_{k}}$ ), and taking the unitaries that mediate the collisions to be partial swap operators. Specifically for all $n$ integer we assume

$$
\hat{U}_{n}=\exp \left[i \theta \hat{\mathbb{S}}_{n}\right]=\cos \theta \mathbb{I}_{n}+i \sin \theta \hat{\mathbb{S}}_{n},
$$

where $\theta \in]-\pi, \pi]$ is a dimensionless parameter that gauges the strength of the collisional event and which is proportional to the collisional time $\delta t$, while $\hat{\mathbb{S}}_{n}=\hat{\mathbb{S}}_{n}^{\dagger}=\hat{\mathbb{S}}_{n}^{-1}$ is the swap operator coupling system A with the $n$th environmental bath subsystem (when acting on states of the form $|\psi\rangle_{\mathrm{A}} \otimes|\phi\rangle_{\mathrm{b}_{n}}$ it exchanges them, i.e., $\left.\hat{\mathbb{S}}_{n}|\psi\rangle_{\mathrm{A}} \otimes|\phi\rangle_{\mathrm{b}_{n}}=|\phi\rangle_{\mathrm{A}} \otimes|\psi\rangle_{\mathrm{b}_{n}}\right)$. Besides implying the property (16) for all input $\hat{\rho}_{\mathrm{A}}^{(0)}$ (this being true [13] as long as $\theta$ is not an integer multiple of $\pi$ ), the choices detailed above ensure that the sum $\hat{H}_{\mathrm{A}}+\sum_{n=1}^{N} \hat{H}_{\mathrm{b}_{n}}$ of the local Hamiltonians of $\mathrm{A}$ and $\mathrm{B}$ commutes with the unitary transformation $\hat{U}_{n} \cdots \hat{U}_{2} \hat{U}_{1}$. Therefore, similarly to the $\Delta E_{\text {int }}=0$ case of the Hamiltonian model, the energy variations of $\mathrm{A}$ in the $\mathrm{CM}$ are compensated by an opposite variation for the bath, leading to the identity $\Delta Q_{\mathrm{A}}^{(n)}=$ $-\Delta Q_{\mathrm{B}}^{(n)}$, where for $\mathrm{X}=\mathrm{A}, \mathrm{B}, \Delta Q_{\mathrm{X}}^{(n)}:=\operatorname{Tr}\left[\hat{H}_{\mathrm{X}}\left(\hat{\rho}_{\mathrm{AB}}^{(n)}-\hat{\rho}_{\mathrm{AB}}^{(0)}\right)\right]$ measures the heat absorbed by the $\mathrm{X}$ system between the zeroth and $n$th step of the process. Using the same arguments adopted in the previous section on the state (14) it is also possible to show that both the Clausius inequality (1) and the extrinsic bound (2) still hold true, i.e.,

$$
\begin{aligned}
& \Delta S_{\mathrm{A}}^{(n)} \geqslant \beta \Delta Q_{\mathrm{A}}^{(n)}, \\
& \Delta S_{\mathrm{A}}^{(n)} \geqslant-\Delta S_{\mathrm{B}}^{(n)},
\end{aligned}
$$

with $\Delta S_{\mathrm{A}}^{(n)}:=S\left(\hat{\rho}_{\mathrm{A}}^{(n)}\right)-S\left(\hat{\rho}_{\mathrm{A}}^{(0)}\right) \quad$ and $\quad \Delta S_{\mathrm{B}}^{(n)}:=S\left(\hat{\rho}_{\mathrm{B}}^{(n)}\right)-$ $S\left(\hat{\rho}_{\mathrm{B}}^{(0)}\right)$ gauging the entropy differences between the zeroth and $n$th step of the process. A detailed derivation of this relation is provided in the Appendix.

Here we only stress that in computing the term on the RHS of (19) we treat B as a real many-body quantum system, retaining all interparticle correlations which may arise between its subenvironment components $b_{1}, b_{2}, \ldots, b_{N}$ due to their collisions with A. According to this choice the explicit evaluation of $\Delta S_{\mathrm{B}}^{(n)}$ is a highly demanding task as it requires the full diagonalization of the density matrix $\hat{\rho}_{\mathrm{B}}^{(n)}$ of the environment. An alternative, and much easier to compute, formulation of this inequality could also be obtained by effectively removing such correlations (e.g., by replacing the joint state of $A B$ that is emerging from the $n$th collision with its reduced factorized counterpart [31]); this yields the following "local" version of 
(a)

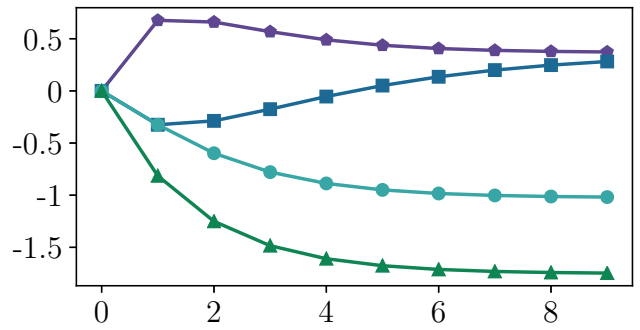

(c)

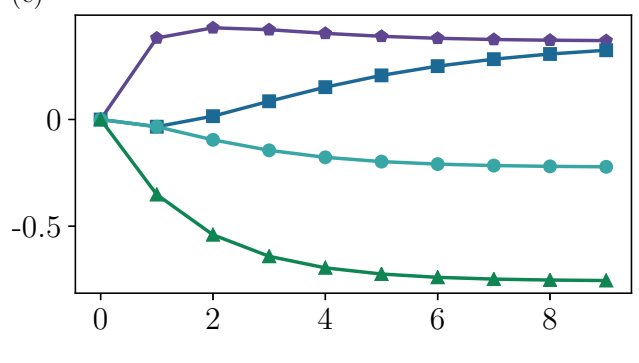

(b)

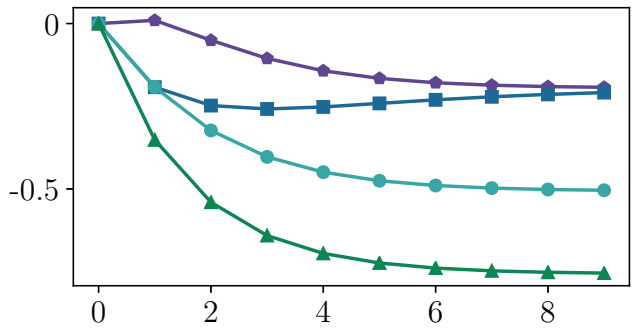

(d)

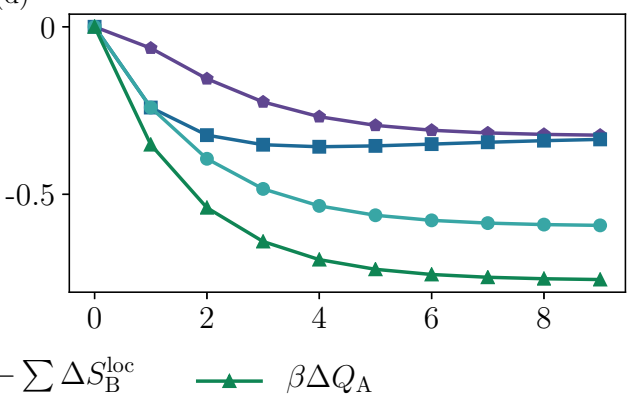

FIG. 1. Numerical evaluation of the bounds for the entropy production in the CM for a qubit system. In the plot the behavior of $\Delta S_{\mathrm{A}}^{(n)}$ and of the quantities $\beta \Delta Q_{\mathrm{A}}^{(n)}$ [intrinsic bound, Eq. (18)], $-\Delta S_{\mathrm{B}}^{(n)}$ [extrinsic bound, Eq. (19)], and $-\Delta S_{\mathrm{B}}^{(n, \text { loc })}$ [local version of the extrinsic bound, Eq. (20)] are shown as a function of the collisional step index $n$ for four different initial states of A represented by the Bloch vectors $\vec{r}^{(0)}=(0,0,1)$ [ground state of A, panel (a)], $(1 / 2,0,0)$ [panel (b)], $(1,0,0)$ [panel (c)], and $(0,0,0)$ [completely mixed state of A, panel (d)], respectively. The reported values fulfill the ordering anticipated in Eq. (21), $-\Delta S_{\mathrm{B}}^{(n)}$ always providing the optimal bound. Furthermore, as $n$ increases, $-\Delta S_{\mathrm{B}}^{(n)}$ approaches $\Delta S_{\mathrm{A}}^{(n)}$ saturating the extrinsic bound (19) in agreement with the asymptotic factorization conjecture of Eq. (23). In all the plots we set $\beta=1$ and $\theta=0.75$ (i.e., below the threshold $\arctan 2$; see Sec. IV A).

the extrinsic bound:

$$
\Delta S_{\mathrm{A}}^{(n)} \geqslant-\Delta S_{\mathrm{B}}^{(n, \mathrm{loc})}
$$

which is provably weaker than the one we present in Eq. (19)—-see the Appendix for details. Another important observation is the fact that, for the CM we analyze here, the identity Eq. (12) still applies. Due to the absence of the $\Delta E_{\mathrm{int}}$ contribution (see discussion above), this implies that for the present model the extrinsic bound (19) and its local counterpart (20) always beat the intrinsic one leading to the following ordering:

$$
\Delta S_{\mathrm{A}}^{(n)} \geqslant-\Delta S_{\mathrm{B}}^{(n)} \geqslant-\Delta S_{\mathrm{B}}^{(n, \text { loc })} \geqslant \beta \Delta Q_{\mathrm{A}}^{(n)} .
$$

As a matter of fact it turns out that the inequality (19) is asymptotically optimal, the gap between $\Delta S_{\mathrm{A}}^{(n)}$ and $-\Delta S_{\mathrm{B}}^{(n)}$ being exponentially decreasing. Introducing the quantum mutual information $I_{\mathrm{A}: \mathrm{B}}^{(n)}:=S\left(\hat{\rho}_{\mathrm{A}}^{(n)}\right)+S\left(\hat{\rho}_{\mathrm{B}}^{(n)}\right)-S\left(\hat{\rho}_{\mathrm{AB}}^{(n)}\right)[23]$ and noticing that $S\left(\hat{\rho}_{\mathrm{AB}}^{(n)}\right)=S\left(\hat{\rho}_{\mathrm{AB}}^{(0)}\right)=S\left(\hat{\rho}_{\mathrm{A}}^{(0)}\right)+S\left(\hat{\rho}_{\mathrm{B}}^{(0)}\right)$ due to unitary invariance of the von Neumann entropy, this result can be equivalently cast in terms of the following identity:

$$
\lim _{n \rightarrow \infty} I_{\mathrm{A}: \mathrm{B}}^{(n)}=0 .
$$

The above expression is a major improvement with respect to the identity (16): while the latter implies that the global state of $\mathrm{A}$ and $\mathrm{B}$ approaches the Gibbs configuration $\hat{\eta}_{\mathrm{A}}^{(\beta)}$ locally on A, Eq. (22) imposes explicit factorization of the joint state in the asymptotic limit of large $n$, i.e.,

$$
\lim _{n \rightarrow \infty} \hat{\rho}_{\mathrm{AB}}^{(n)}=\hat{\eta}_{\mathrm{A}}^{(\beta)} \otimes \hat{\Lambda}_{\mathrm{B}}
$$

with the density matrix $\hat{\Lambda}_{\mathrm{B}}$ maintaining a functional dependence upon the input state $\hat{\rho}_{\mathrm{A}}^{(0)}$ of $\mathrm{A}$ as a consequence of the unitary mapping (14). For $|\theta| \geqslant \arctan 2 \simeq 1.107$ (strongcollisions regime) an explicit proof of the asymptotic factorization property (22) is presented in Sec. IV: it works for any finite-dimensional system $\mathrm{A}$ and for all choices of the inverse temperature $\beta$. For lower values of $|\theta|$ our argument fails; still we conjecture that (23) should hold also in those cases. In support of this conjecture we present some numerical evidences where we have tested the model for the case where A and the subsystems $b_{n}$ are qubits with local Hamiltonians $\hat{H}_{\mathrm{A}}=\frac{1}{2} \hat{\sigma}_{\mathrm{A}}^{(3)}$ and $\hat{H}_{\mathrm{b}}=\frac{1}{2} \hat{\sigma}_{\mathrm{b}}^{(3)}, \hat{\sigma}^{(3)}$ being the third Pauli operator and the energy scale being measured in units $\hbar \omega=1$. Results are reported in Sec. III and summarized in Figs. 1 and 2.

\section{Qubit case}

Here we study in detail the CM for the special case where $A$ and the subenvironments $\mathrm{b}$ are qubit systems. Adopting the Bloch sphere representation we write the state $\hat{\rho}_{\mathrm{A}}^{(n)}$ of A after the $n$th collision

$$
\hat{\rho}_{\mathrm{A}}^{(n)}=\frac{\hat{\mathbb{I}}_{\mathrm{A}}+\vec{r}^{(n)} \cdot \vec{\sigma}_{\mathrm{A}}}{2},
$$



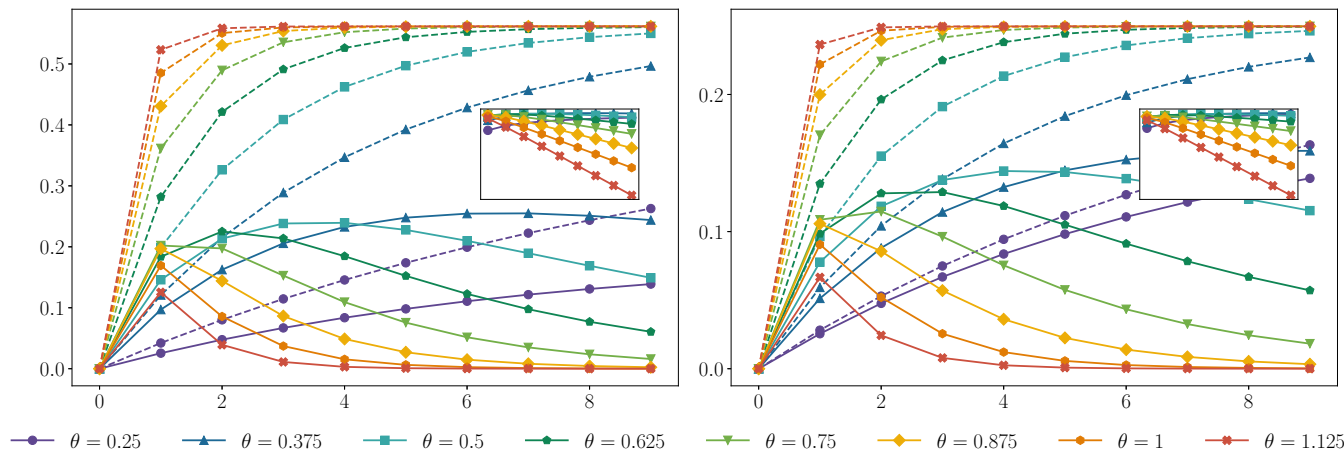

FIG. 2. Numerical evaluation of the intrinsic (18) and extrinsic (19) bounds for the entropy production in the CM for a qubit system. Dashed lines show the behavior of $\Delta S_{\mathrm{A}}^{(n)}-\beta \Delta Q_{\mathrm{A}}^{(n)}$ as a function of the collisional step $n$ for various values of the partial swap parameter $\theta$ of Eq. (17). Notice that as expected for Markovian processes this is an increasing function of $n$, which saturates to the value $S\left(\hat{\rho}_{\mathrm{A}}^{(0)} \| \hat{\eta}_{\mathrm{A}}^{(\beta)}\right)$ in agreement with (10). The continuous lines represent instead the behavior of $\Delta S_{\mathrm{A}}^{(n)}+\Delta S_{\mathrm{B}}^{(n)}$. In this case the function has no monotonic behavior and as $n$ increases it tends to nullify in agreement with the factorization prediction of Eqs. (22) and (23). In the inset the same quantity is shown in logarithmic scale. In all plots the input state of $\mathrm{A}$ is associated to $\vec{r}^{(0)}=(1 / 2,0,0)$, while the temperature of the bath is such that $\beta=1$ in the left panel and $\beta=0.5$ in the right panel.

with $\hat{\mathbb{I}}_{\mathrm{A}}$ and $\vec{\sigma}_{\mathrm{A}}=\left(\hat{\sigma}_{\mathrm{A}}^{(1)}, \hat{\sigma}_{\mathrm{A}}^{(2)}, \hat{\sigma}_{\mathrm{A}}^{(3)}\right)$ being respectively the identity and Pauli vector operators. From this we can compute the associated entropy $S\left(\hat{\rho}_{\mathrm{A}}^{(n)}\right)$ and the mean energy of the state as

$$
\begin{gathered}
S\left(\hat{\rho}_{\mathrm{A}}^{(n)}\right)=H_{2}\left(\frac{1+\left|\vec{r}^{(n)}\right|}{2}\right), \\
E_{\mathrm{A}}^{(n)}:=\operatorname{Tr}\left[\hat{H}_{\mathrm{A}} \hat{\rho}_{\mathrm{A}}^{(n)}\right]=r_{3}^{(n)} / 2,
\end{gathered}
$$

where $H_{2}(x):=-x \ln x-(1-x) \ln (1-x)$ is the Shannon binary entropy functional and where we have used the fact that $\hat{H}_{\mathrm{A}}=\frac{1}{2} \hat{\sigma}_{\mathrm{A}}^{(3)}$. Accordingly, introducing the Bloch vector $\vec{r}^{(0)}$ of the input state $\hat{\rho}_{\mathrm{A}}^{(0)}$ of $\mathrm{A}$, we can then write

$$
\begin{gathered}
\Delta S_{\mathrm{A}}^{(n)}=H_{2}\left(\frac{1+\left|\vec{r}^{(n)}\right|}{2}\right)-H_{2}\left(\frac{1+\left|\vec{r}^{(0)}\right|}{2}\right), \\
\Delta Q_{\mathrm{A}}^{(n)}=\left(r_{3}^{(n)}-r_{3}^{(0)}\right) / 2 .
\end{gathered}
$$

From the definition of $H_{2}(x)$ it follows that $\Delta S_{\mathrm{A}}^{(n)}$ is an increasing function of the length $\left|\vec{r}^{(n)}\right|$ of the Bloch vector $\vec{r}^{(n)}$, while $\Delta Q_{\mathrm{A}}^{(n)}$ is just linearly dependent upon the $z$-axis component of such vector.

A closed expression for these quantities can then be obtained by exploiting the properties of the partial swap transformation (17), to recast (15) into the following recursive mapping:

$$
\vec{r}^{(n)}=\cos ^{2} \theta \vec{r}^{(n-1)}+\sin ^{2} \theta \vec{s},
$$

with $\vec{s}$ being the Bloch vector associated with the input state $\hat{\eta}_{\mathrm{b}}^{(\beta)}=e^{-\beta \hat{\sigma}_{\mathrm{b}}^{(3)} / 2} / Z_{\mathrm{b}}(\beta)$ of the environmental subsystem $\mathrm{b}$, i.e.,

$$
\vec{s}=(0,0, s), \quad s:=s(\beta)=-\tanh (\beta / 2) .
$$

Iterating, expression (29) can be formally integrated, giving

$$
\begin{aligned}
\vec{r}^{(n)} & =\cos ^{2 n} \theta \vec{r}^{(0)}+\left(1-\cos ^{2 n} \theta\right) \vec{s} \\
& =\vec{s}+\cos ^{2 n} \theta \Delta \vec{r}^{(0)},
\end{aligned}
$$

with $\Delta \vec{r}^{(0)}:=\vec{r}^{(0)}-\vec{s}$ the difference between the Bloch vector $\vec{r}^{(0)}$ of the input state of A and $\vec{s}$. The length and the $z$-axis component of the vector (31) can then be computed as

$$
\begin{gathered}
\left|\vec{r}^{(n)}\right|=\sqrt{s^{2}+\cos ^{4 n} \theta\left|\Delta \vec{r}^{(0)}\right|^{2}+\cos ^{2 n} \theta s \Delta r_{3}^{(0)}}, \\
r_{3}^{(n)}-r_{3}^{(0)}=\left(\cos ^{2 n} \theta-1\right) \Delta r_{3}^{(0)} .
\end{gathered}
$$

By the same token we can now compute the local output states of the environmental subsystems $\hat{\rho}_{\mathrm{b}}^{(n)}=$ $\operatorname{Tr}_{\mathrm{A}}\left[\hat{U}\left(\hat{\rho}_{\mathrm{A}}^{(n-1)} \otimes \hat{\eta}_{\mathrm{b}}^{(\beta)}\right) \hat{U}^{\dagger}\right]$, which via Eq. (A4) provide a lower bound to $\Delta S_{\mathrm{A}}^{(n)}$. Following the same derivation given above the Bloch vector of such state can be expressed as

$$
\begin{aligned}
\vec{s}^{(n)} & =\sin ^{2} \theta \vec{r}^{(n-1)}+\cos ^{2} \theta \vec{s} \\
& =\vec{s}+\sin ^{2} \theta \cos ^{2(n-1)} \theta \Delta \vec{r}^{(0)},
\end{aligned}
$$

where the last identity follows from (31). The length of such vector is hence

$$
\begin{aligned}
\left|\vec{s}^{(n)}\right|= & \left(s^{2}+\sin ^{4} \theta \cos ^{4(n-1)} \theta\left|\Delta \vec{r}^{(0)}\right|^{2}\right. \\
& \left.+\sin ^{2} \theta \cos ^{2(n-1)} \theta s \Delta r_{3}^{(0)}\right)^{1 / 2},
\end{aligned}
$$

yielding

$$
\Delta S_{\mathrm{B}}^{(\mathrm{loc})}=\sum_{k=1}^{n} H_{2}\left(\frac{1+\left|\vec{s}^{(k)}\right|}{2}\right)-n H_{2}\left(\frac{1+s}{2}\right)
$$

for the quantity (A4) of the Appendix that defines the local version (20) of the extrinsic bound.

Expressions (27), (28), and (36) are used for the plots of Figs. 1 and 2. The evaluation of $\Delta S_{\mathrm{B}}^{(n)}$ instead requires a complete diagonalization of the many-body quantum state of the environment $\mathrm{B}$.

\section{CORRELATIONS DECAY}

This section focuses on the factorization property (23). As a preliminary observation we notice that, irrespective of the values of $\theta$ and $\beta$, Eq. (23) is trivially verified when the input 
state of $\mathrm{A}$ is already at thermal equilibrium, i.e., $\hat{\rho}_{\mathrm{A}}^{(0)}=\hat{\eta}_{\mathrm{A}}^{(\beta)}$. Indeed, under this condition, for all $n$ one has:

$$
\hat{\rho}_{\mathrm{AB}}^{(n)}=\hat{\eta}_{\mathrm{A}}^{(\beta)} \otimes\left(\hat{\eta}_{\mathrm{b}}^{(\beta)}\right)^{\otimes N},
$$

due to the fact that, given $\widehat{\mathbb{S}}$ the swap operator acting on the Hilbert space $\mathcal{H}^{\otimes 2}$, the state $\hat{\eta} \otimes \hat{\eta}$ commutes with it (i.e., $[\hat{\mathbb{S}}, \hat{\eta} \otimes \hat{\eta}]=0)$, and hence with $e^{i \theta \hat{\mathbf{S}}}$, thus leading to $e^{i \theta \hat{\mathbb{S}}}(\hat{\eta} \otimes$ $\hat{\eta}) e^{-i \theta \hat{\mathbb{S}}}=\hat{\eta} \otimes \hat{\eta}$. Also, Eq. (23) can be easily shown to hold for arbitrary inputs, in the case where the bath temperature is zero $(\beta \rightarrow \infty)$ and the local Hamiltonians of the model have a nondegenerate ground state $|0\rangle$. In this case in fact the Gibbs states $\hat{\eta}_{\mathrm{b}}^{(\beta)}$ correspond to the pure vectors $|0\rangle_{\mathrm{b}}$, while (16) yields

$$
\lim _{n \rightarrow \infty} \hat{\rho}_{\mathrm{A}}^{(n)}=|0\rangle_{\mathrm{A}}\langle 0|,
$$

which can only be fulfilled by having a joint state $\hat{\rho}_{\mathrm{AB}}^{(n)}$ that asymptotically approaches a state of the form $|0\rangle_{\mathrm{A}}\langle 0| \otimes \hat{\Lambda}_{\mathrm{B}}$.

In Sec. IV A we shall prove that (23) holds for the CM model as long as the strength of the partial swapping is sufficiently large. Instead in Sec. IV B the factorization property (23) is shown to hold for a slight modification of the scheme where we do alternate sequences of collisions with a full dephasing process on $\mathrm{A}$.

\section{A. Factorization proof for the strong-collisions regime}

Consider now the nontrivial case where $\hat{\rho}_{\mathrm{A}}^{(0)} \neq \hat{\eta}_{\mathrm{A}}^{(\beta)}$ and $\beta$ is finite. In order to prove the identity (23) we notice that Eq. (14) which defines the joint state of AB after $n$ collisions implies the following recursive formula $\hat{\rho}_{\mathrm{AB}}^{(n)}=\mathcal{U}_{n}\left[\hat{\rho}_{\mathrm{AB}}^{(n-1)}\right]$. We now express such state as the sum of two terms

$$
\hat{\rho}_{\mathrm{AB}}^{(n)}=\hat{R}_{\mathrm{AB}}^{(n)}+\hat{T}_{\mathrm{AB}}^{(n)},
$$

with $\hat{R}_{\mathrm{AB}}^{(n)}$ representing the contribution where A factorizes from $\mathrm{B}$ and is in $\hat{\eta}_{\mathrm{A}}^{(\beta)}$ (i.e., $\hat{R}_{\mathrm{AB}}^{(n)}=\hat{\eta}_{\mathrm{A}}^{(\beta)} \otimes \hat{R}_{\mathrm{B}}^{(n)}$ ), while $\hat{T}_{\mathrm{AB}}^{(n)}$ contains all the remaining ones. An explicit derivation of such decomposition can be formally derived via the following construction: for $n=0$, using the fact that $\hat{\rho}_{\mathrm{A}}^{(0)} \neq \hat{\eta}_{\mathrm{A}}^{(\beta)}$ we set $\hat{R}_{\mathrm{AB}}^{(0)}=0$ and $\hat{T}_{\mathrm{AB}}^{(0)}=\hat{\rho}_{\mathrm{AB}}^{(0)}$. Then for $n=1$ we use Eq. (17) and the properties of the swap operator to write

$$
\begin{aligned}
\hat{R}_{\mathrm{AB}}^{(1)} & =\sin ^{2} \theta\left(\hat{\mathbb{S}}_{1} \hat{\rho}_{\mathrm{AB}}^{(0)} \hat{\mathbb{S}}_{1}\right) \\
& =\sin ^{2} \theta\left(\hat{\eta}_{\mathrm{A}}^{(\beta)} \otimes \hat{\rho}_{\mathrm{b}_{1}}^{(0)} \otimes\left[\hat{\eta}_{\mathrm{b}}^{(\beta)}\right]^{\otimes N-1}\right), \\
\hat{T}_{\mathrm{AB}}^{(1)} & =\cos ^{2} \theta \hat{\rho}_{\mathrm{AB}}^{(0)}+i \sin \theta \cos \theta\left[\hat{\mathbb{S}}_{1}, \hat{\rho}_{\mathrm{AB}}^{(0)}\right],
\end{aligned}
$$

where $[\cdots, \cdots]$ stands for the commutator. For higher values of $n$ we can derive a recursive formula connecting $\hat{R}_{\mathrm{AB}}^{(n+1)}$, $\hat{T}_{\mathrm{AB}}^{(n+1)}$ to $\hat{R}_{\mathrm{AB}}^{(n)}, \hat{T}_{\mathrm{AB}}^{(n)}$, by noticing that

$$
\mathcal{U}_{n+1}\left[\hat{R}_{\mathrm{AB}}^{(n)}\right]=\hat{R}_{\mathrm{AB}}^{(n)},
$$

which follows once more from the fact that states $\hat{\eta} \otimes \hat{\eta}$ are invariant under partial swaps (see argument at the beginning of the section). Therefore, the only part of $\mathcal{U}_{n+1}\left[\hat{T}_{\mathrm{AB}}^{(n)}\right]$ that contributes to $\hat{R}_{\mathrm{AB}}^{(n+1)}$ is the one which has $\hat{\mathbb{S}}_{n+1}$ either only on the right and or only on the left. Accordingly, we have

$$
\begin{gathered}
\hat{R}_{\mathrm{AB}}^{(n+1)}=\hat{R}_{\mathrm{AB}}^{(n)}+\sin ^{2} \theta \hat{\mathbb{S}}_{n+1} \hat{T}_{\mathrm{AB}}^{(n)} \hat{\mathbb{S}}_{n+1}, \\
\hat{T}_{\mathrm{AB}}^{(n+1)}=\cos ^{2} \theta \hat{T}_{\mathrm{AB}}^{(n)}+i \sin \theta \cos \theta\left[\hat{\mathbb{S}}_{n+1}, \hat{T}_{\mathrm{AB}}^{(n)}\right] .
\end{gathered}
$$

Exploiting the subadditivity of the norm we then get

$$
\begin{aligned}
\left\|\hat{T}_{\mathrm{AB}}^{(n+1)}\right\| & \leqslant\left|\cos ^{2} \theta\right|\left\|\hat{T}_{\mathrm{AB}}^{(n)}\right\|+|\sin \theta \cos \theta|\left\|\left[\hat{\mathbb{S}}_{n+1}, \hat{T}_{\mathrm{AB}}^{(n)}\right]\right\| \\
& \leqslant\left(\left|\cos ^{2} \theta\right|+2|\sin \theta \cos \theta|\right)\left\|\hat{T}_{\mathrm{AB}}^{(n)}\right\|
\end{aligned}
$$

where in the second line we used the fact that $\hat{\mathrm{S}}_{n+1}$ is unitary to claim that $\left\|\hat{\mathrm{S}}_{n+1} \hat{T}_{\mathrm{AB}}^{(n)}\right\|=\left\|\hat{T}_{\mathrm{AB}}^{(n)}\right\|$ (the above results being true in any operator norm). Iterating this we can then write

$$
\left\|\hat{T}_{\mathrm{AB}}^{(n+1)}\right\| \leqslant\left(\left|\cos ^{2} \theta\right|+2|\sin \theta \cos \theta|\right)^{n+1}\left\|\hat{\rho}_{\mathrm{AB}}^{(0)}\right\|,
$$

where we used the fact that $\hat{T}_{\mathrm{AB}}^{(0)}=\hat{\rho}_{\mathrm{AB}}^{(0)}$. Now we observe that, for

$$
|\theta|>\arctan 2,
$$

we have $\left|\cos ^{2} \theta\right|+2|\sin \theta \cos \theta|<1$ and hence from (46)

$$
\lim _{n \rightarrow \infty}\left\|\hat{T}_{\mathrm{AB}}^{(n)}\right\|=0,
$$

implying that in the large $n$ limit $\hat{T}_{\mathrm{AB}}^{(n)}$ approaches zero allowing us to identify $\hat{\rho}_{\mathrm{AB}}^{(n)}$ with $\hat{R}_{\mathrm{AB}}^{(n)}$ as required by Eq. (23).

\section{B. Asymptotic factorization for $\mathrm{CM}$ with a little help from full dephasing on A}

Here we show that the asymptotic factorization property (23) can be proven under a slight modification of the CM where, instead of letting $\mathrm{A}$ and $\mathrm{B}$ evolve under a sequence of collisional events as in (14) every $k \gg 1$ collisions we force A to undergo full dephasing transformation $\mathcal{D}_{\mathrm{A}}$, which destroys all its off-diagonal elements with respect to the local energy eigenbasis $\left\{|j\rangle_{\mathrm{A}}\right\}$, i.e.,

$$
\mathcal{D}_{\mathrm{A}}\left[|j\rangle_{\mathrm{A}}\left\langle j^{\prime}\right|\right]=\delta_{j, j^{\prime}}|j\rangle_{\mathrm{A}}\langle j|,
$$

with $\delta_{j, j^{\prime}}$ the Kronecker delta. For the sake of simplicity we present this argument for the special case of A being a qubit, but the same can be generalized to arbitrary dimensions. Also we stress that, as long as the property (16) is verified together with the assumption that the state of $\hat{\eta}_{\mathrm{A}}^{(\beta)}$ will not evolve during a collisional event, the derivation we present below does not rely on the specific form of the unitaries given in Eq. (17).

Let us hence divide the subsystems of B into groups of $k$ elements: the set $\mathrm{B}_{1}$, containing the first $k$ subenvironments, the set $\mathrm{B}_{2}$ containing the second $k$ subenvironments, and so on and so forth. Take then the joint state of $\mathrm{A}_{\text {and }} \mathrm{B}_{1}$ after the first $k$ unitary collisions have been performed, and expand it with respect to the local energy basis of $\mathrm{A}$, i.e.,

$$
\begin{aligned}
\hat{\rho}_{\mathrm{AB}_{1}}^{(k)} & =\mathcal{U}_{k} \circ \cdots \circ \mathcal{U}_{2} \circ \mathcal{U}_{1}\left[\hat{\rho}_{\mathrm{A}}^{(0)} \otimes\left(\hat{\eta}_{\mathrm{b}}^{(\beta)}\right)^{\otimes k}\right] \\
& =\sum_{j, j^{\prime}}|j\rangle_{\mathrm{A}}\left\langle j^{\prime}\right| \otimes \hat{\Pi}_{\mathrm{B}_{1}}^{\left(j, j^{\prime}\right)}\left(\rho_{\mathrm{A}}^{(0)}\right),
\end{aligned}
$$

with $\hat{\Pi}_{\mathrm{B}_{1}}^{\left(j, j^{\prime}\right)}\left(\rho_{\mathrm{A}}^{(0)}\right):={ }_{\mathrm{A}}\left\langle j^{\prime}\left|\hat{\rho}_{\mathrm{AB}}^{(k)}\right| j\right\rangle_{\mathrm{A}}$ being operators of $\mathrm{B}_{1}$ which inherit a linear dependence upon the input state $\hat{\rho}_{\mathrm{A}}^{(0)}$ 
of A. Taking the partial trace with respect to $B_{1}$ this yields the density operator

$$
\hat{\rho}_{\mathrm{A}}^{(k)}=\sum_{j, j^{\prime}} M_{j, j^{\prime}}^{(k)}\left(\rho_{\mathrm{A}}^{(0)}\right)|j\rangle_{\mathrm{A}}\left\langle j^{\prime}\right|
$$

with matrix coefficients

$$
M_{j, j^{\prime}}^{(k)}\left(\rho_{\mathrm{A}}^{(0)}\right):=\operatorname{Tr}_{\mathrm{B}_{1}}\left[\hat{\Pi}_{\mathrm{B}_{1}}^{\left(j, j^{\prime}\right)}\left(\rho_{\mathrm{A}}^{(0)}\right)\right] .
$$

From (16) we know that for large $k$ should approach the stationary configuration $\hat{\eta}_{\mathrm{A}}^{(\beta)}$, which by construction, is diagonal with respect to the basis $\left\{|j\rangle_{\mathrm{A}}\right\}$ with eigenvalues equal to $\eta_{j}^{(\beta)}=e^{-\beta \hbar E_{j}} / Z(\beta)$. Accordingly, for any given positive $\epsilon<1$, we can choose $k$ sufficiently large to guarantee that the following inequalities hold:

$$
\begin{gathered}
\left|M_{j, j}^{(k)}\left(\rho_{\mathrm{A}}^{(0)}\right)-\eta_{j}^{(\beta)}\right|<\epsilon, \\
\left|M_{j, j^{\prime}}^{(k)}\left(\rho_{\mathrm{A}}^{(0)}\right)\right|<\epsilon, \quad \forall j \neq j^{\prime} .
\end{gathered}
$$

If we next apply the full dephasing (49) to the state (50) we get

$$
\hat{\rho}_{\mathrm{AB}}^{(k)} \rightarrow|0\rangle_{\mathrm{A}}\left\langle 0\left|\otimes \hat{\Pi}_{\mathrm{B}_{1}}^{(0,0)}\left(\rho_{\mathrm{A}}^{(0)}\right)+\right| 1\right\rangle_{\mathrm{A}}\langle 1| \otimes \hat{\Pi}_{\mathrm{B}_{1}}^{(1,1)}\left(\rho_{\mathrm{A}}^{(0)}\right),
$$

where, for the first time, we explicitly used the fact that system A is a qubit. Summing and subtracting the term $\frac{\eta_{0}^{(\beta)}}{\eta_{1}^{(\beta)}} \hat{\Pi}_{\mathrm{B}_{1}}^{(1,1)}\left(\rho_{\mathrm{A}}^{(0)}\right)$ and using the definition of $\hat{\eta}_{\mathrm{A}}^{(\beta)}$ the RHS of this expression can thus be rewritten as

$$
|0\rangle_{\mathrm{A}}\langle 0| \otimes \hat{\Delta}_{\mathrm{B}_{1}}^{(k)}\left(\rho_{\mathrm{A}}^{(0)}\right)+\hat{\eta}_{\mathrm{A}}^{(\beta)} \otimes \hat{\Xi}_{\mathrm{B}_{1}}\left(\rho_{\mathrm{A}}^{(0)}\right),
$$

with $\hat{\Xi}_{\mathrm{B}_{1}}\left(\rho_{\mathrm{A}}^{(0)}\right):=\hat{\Pi}_{\mathrm{B}_{1}}^{(1,1)}\left(\rho_{\mathrm{A}}^{(0)}\right) / \eta_{1}^{(\beta)}$ and with

$$
\hat{\Delta}_{\mathrm{B}_{1}}^{(k)}\left(\rho_{\mathrm{A}}^{(0)}\right):=\hat{\Pi}_{\mathrm{B}_{1}}^{(0,0)}\left(\rho_{\mathrm{A}}^{(0)}\right)-\frac{\eta_{0}^{(\beta)}}{\eta_{1}^{(\beta)}} \hat{\Pi}_{\mathrm{B}_{1}}^{(1,1)}\left(\rho_{\mathrm{A}}^{(0)}\right),
$$

an operator whose trace norm $\left\|\hat{\Delta}_{\mathrm{B}_{1}}^{(k)}\left(\rho_{\mathrm{A}}^{(0)}\right)\right\|_{1}$, for sufficiently large $k$, can be forced to be strictly smaller than one thanks to (53). Indeed summing and subtracting $\frac{M_{0,0}^{(k)}\left(\rho_{\mathrm{A}}^{(0)}\right)}{M_{1,1}^{(k)}\left(\rho_{\mathrm{A}}^{(0)}\right)} \hat{\Pi}_{\mathrm{B}_{1}}^{(1,1)}\left(\rho_{\mathrm{A}}^{(0)}\right)$ to $\hat{\Delta}_{\mathrm{B}_{1}}^{(k)}\left(\rho_{\mathrm{A}}^{(0)}\right)$ and using the triangular inequality of the trace norm we can write $\left\|\hat{\Delta}_{\mathrm{B}_{1}}^{(k)}\left(\rho_{\mathrm{A}}^{(0)}\right)\right\|_{1} \leqslant \alpha^{(k)}+\beta^{(k)}$ with

$$
\begin{aligned}
& \alpha^{(k)}:=M_{0,0}^{(k)}\left(\rho_{\mathrm{A}}^{(0)}\right)\left\|\frac{\hat{\Pi}_{\mathrm{B}_{1}}^{(0,0)}\left(\rho_{\mathrm{A}}^{(0)}\right)}{M_{0,0}^{(k)}\left(\rho_{\mathrm{A}}^{(0)}\right)}-\frac{\hat{\Pi}_{\mathrm{B}}^{(1,1)}\left(\rho_{\mathrm{A}}^{(0)}\right)}{M_{1,1}^{(k)}\left(\rho_{\mathrm{A}}^{(0)}\right)}\right\|_{1}, \\
& \beta^{(k)}:=\left|M_{0,0}^{(k)}\left(\rho_{\mathrm{A}}^{(0)}\right)-\frac{\eta_{0}^{(\beta)}}{\eta_{1}^{(\beta)}} M_{1,1}^{(k)}\left(\rho_{\mathrm{A}}^{(0)}\right)\right|\left\|\frac{\hat{\Pi}_{\mathrm{B}}^{(1,1)}\left(\rho_{\mathrm{A}}^{(0)}\right)}{M_{1,1}^{(k)}\left(\rho_{\mathrm{A}}^{(0)}\right)}\right\|_{1} .
\end{aligned}
$$

The thesis then follows by noticing that, for sufficiently large $k$, we can ensure that $\alpha^{(k)}$ is a quantity smaller than 1 with $\beta^{(k)}$ being arbitrarily small. Regarding $\alpha^{(k)}$ this can be shown by exploiting the fact that in the limit of high $k, M_{0,0}^{(k)}\left(\rho_{\mathrm{A}}^{(0)}\right)$ approaches $\eta_{0}^{(\beta)}$, which for $\beta>0$ is always strictly smaller than one, while, since $\frac{\hat{\Pi}_{B_{1}}^{(0,0)}\left(\rho_{A}^{(0)}\right)}{M_{0,0}^{(k)}\left(\rho_{\mathrm{A}}^{(0)}\right)}$ and $\frac{\hat{\Pi}_{B_{1}^{(1,1)}}^{(0)}\left(\rho_{\mathrm{A}}^{(0)}\right)}{M_{1,1}^{(k)}\left(\rho_{\mathrm{A}}^{(0)}\right)}$ are properly normalized density matrices the norm of their difference is certainly smaller than or equal to one. Regarding $\beta^{(k)}$ instead we can use (53) to show that $\left|M_{0,0}^{(k)}\left(\rho_{\mathrm{A}}^{(0)}\right)-\frac{\eta_{0}^{(\beta)}}{\eta_{1}^{(\beta)}} M_{1,1}^{(k)}\left(\rho_{\mathrm{A}}^{(0)}\right)\right|$ approach zero for large values of $k$, while $\left\|\frac{\hat{\Pi}_{B_{1}}^{(1,1)}\left(\rho_{A}^{(0)}\right)}{M_{1,1}^{(k)}\left(\rho_{\mathrm{A}}^{(0)}\right)}\right\|_{1}=1$ because it is the trace norm of a properly normalized state.

Equation (55) represents the state of the $\mathrm{AB}_{1}$ after $k$ unitary collisions and a single dephasing event $\mathcal{D}_{\mathrm{A}}$. We now repeat the full procedure introducing the second $k$ subsystems of $B$, i.e., the elements of the subset $B_{2}$. We notice the part of the state (55) which has A already in $\hat{\eta}_{\mathrm{A}}^{(\beta)}$ does not evolve. The only element that undergoes modification is the first component of the state. Iterating the above procedure we hence arrive at

$$
|0\rangle_{\mathrm{A}}\langle 0| \otimes \hat{\Delta}_{\mathrm{B}_{1}}^{(k)}\left(\rho_{\mathrm{A}}^{(0)}\right) \otimes \hat{\Delta}_{\mathrm{B}_{2}}^{(k)}\left(|0\rangle_{\mathrm{A}}\right)+\hat{\eta}_{\mathrm{A}}^{(\beta)} \otimes \hat{\Xi}_{\mathrm{B}_{1} \mathrm{~B}_{2}}\left(\rho_{\mathrm{A}}^{(0)}\right),
$$

with $\hat{\Delta}_{\mathrm{B}_{2}}^{(k)}\left(|0\rangle_{\mathrm{A}}\right)$ as in (57) for $\rho_{\mathrm{A}}^{(0)}=|0\rangle_{\mathrm{A}}\langle 0|$ and $\hat{\Xi}_{\mathrm{B}_{1} \mathrm{~B}_{2}}\left(\rho_{\mathrm{A}}^{(0)}\right)$ a proper operator of $\mathrm{B}_{1} \mathrm{~B}_{2}$. By the same token after $q$ of such steps we get

$$
\begin{aligned}
& |0\rangle_{\mathrm{A}}\langle 0| \otimes \hat{\Delta}_{\mathrm{B}_{1}}^{(k)}\left(\rho_{\mathrm{A}}^{(0)}\right) \otimes_{\ell=2}^{q}\left(\hat{\Delta}_{\mathrm{B}_{\ell}}^{(k)}\left(|0\rangle_{\mathrm{A}}\right)\right) \\
& \quad+\hat{\eta}_{\mathrm{A}}^{(\beta)} \otimes \hat{\Xi}_{\mathrm{B}_{1} \mathrm{~B}_{2} \cdots \mathrm{B}_{\mathrm{q}}}\left(\rho_{\mathrm{A}}^{(0)}\right) .
\end{aligned}
$$

Notice that the first contribution has a trace norm which is equal to $\||0\rangle_{\mathrm{A}}\left\langle\left. 0\right|_{1} \otimes \hat{\Delta}_{\mathrm{B}_{1}}^{(k)}\left(\rho_{\mathrm{A}}^{(0)}\right) \otimes_{\ell=2}^{q}\left(\hat{\Delta}_{\mathrm{B}_{\ell}}^{(k)}\left(|0\rangle_{\mathrm{A}}\right)\right) \|_{1}=\right.$ $\left\|\hat{\Delta}_{\mathrm{B}_{1}}^{(k)}\left(\rho_{\mathrm{A}}^{(0)}\right)\right\| \| \hat{\Delta}_{\mathrm{B}_{\ell}}^{(k)}\left(|0\rangle_{\mathrm{A}}\right) \|_{1}^{q-1}$ and hence is exponentially decreasing in $q$. Accordingly we can claim that for large $q$ the state of $\mathrm{AB}$ will be determined by the second contribution which explicitly factorizes as in Eq. (23).

\section{CONCLUSIONS}

The scheme of Ref. [13] is arguably the simplest thermalization model one can analyze which, within the assumptions of the CM approach, appears to be consistent both thermodynamically and from the point of view of open quantum dynamics. Our analysis clarifies that in this context the Clausius inequality is always outperformed by the extrinsic bound that relates $\Delta S_{\mathrm{A}}$ to the entropy increment of the thermal environment $\mathrm{B}$.

Most interesting, in the limit of infinitely many collisions, the latter turns out to be asymptotically optimal, indicating that the model induces a complete factorization of $\mathrm{A}$ from B. To our understanding, this progressive factorization arises as the result of the balance of two competing effects that take place at each swapping collision: on one hand, every interaction with a new ancilla tends, in principle, to establish new correlations between the environment and the system. On the other hand, this same interaction tends to reduce the correlations the system established with the previous ancillas, by transforming them into intraenvironment correlations via partial replacement of the system degree of freedom with those of the new ancilla due to the action of the swap gate. As a matter of fact the first mechanism becomes more and more feeble approaching the fixed point of the evolution.

We have shown that such asymptotic factorization holds true at least when the strength of the collision is sufficient large: also in view of our numerical analysis, we suspect however that this result should be fairly general and we plan to further investigate it in the future. 


\section{ACKNOWLEDGMENT}

A.D.P. acknowledges financial support from the University of Florence in the framework of the University Strategic Project Program 2015 (Project No. BRS00215).

\section{APPENDIX: EXPLICIT DERIVATION OF THE ENTROPIC BOUNDS FOR THE CM}

The global form (18) of the Clausius inequality is obtained by applying the relative-entropy monotonicity argument to the states $\hat{\rho}_{\mathrm{A}}^{(n)}$ and $\hat{\eta}_{\mathrm{A}}^{(\beta)}$. The incremental version of this follows instead by using the same procedure by comparing the entropies of $\hat{\rho}_{\mathrm{A}}^{(n)}$ and $\hat{\rho}_{\mathrm{A}}^{(n-1)}$ via Eq. (15), obtaining the inequality

$$
d S_{\mathrm{A}}^{(n)} \geqslant \beta d Q_{\mathrm{A}}^{(n)},
$$

with $\quad d S_{\mathrm{A}}^{(n)}:=S\left(\hat{\rho}_{\mathrm{A}}^{(n)}\right)-S\left(\hat{\rho}_{\mathrm{A}}^{(n-1)}\right) \quad$ and $\quad d Q_{\mathrm{A}}^{(n)}:=\operatorname{Tr}\left[\hat{H}_{A}\right.$ $\left.\left(\hat{\rho}_{\mathrm{A}}^{(n)}-\hat{\rho}_{\mathrm{A}}^{(n-1)}\right)\right]$.

The extrinsic bound (19) is obtained by invoking the subadditivity of the von Neumann entropy of the density matrix (14): here $\Delta S_{\mathrm{B}}^{(n)}$ represents the global entropy gain of the multipartite bath $\mathrm{B}$, which properly accounts for all possible correlations between its constituents $b_{1}, b_{2}, \ldots, b_{N}$. Writing it explicitly results in the following expression:

$$
\Delta S_{\mathrm{A}}^{(n)} \geqslant-\Delta S_{\mathrm{B}}^{(n)}=n S\left(\hat{\eta}_{\mathrm{b}}^{(\beta)}\right)-S\left(\hat{\rho}_{B}^{(n)}\right),
$$

where we used the fact that at the beginning of the A-B interactions the bath is described by the factorized state where all its constituents are initialized into the same Gibbs state $\hat{\eta}_{\mathrm{b}}^{(\beta)}$. An incremental version of this inequality instead follows by using the same technique applied to the state (15), i.e.,

$$
d S_{\mathrm{A}}^{(n)} \geqslant-d S_{\mathrm{b}}^{(n)}=S\left(\hat{\eta}_{\mathrm{b}}^{(\beta)}\right)-S\left(\hat{\rho}_{\mathrm{b}}^{(n)}\right)
$$

Here $d S_{\mathrm{b}}^{(n)}$ represents the local entropy variation of the $n$th environmental subsystem $\mathrm{b}_{n}$ after its collision with A (by construction such system evolves from $\hat{\eta}_{\mathrm{b}}^{(\beta)}$ to
$\left.\hat{\rho}_{\mathrm{b}}^{(n)}:=\operatorname{Tr}_{\mathrm{A}}\left[\hat{U}\left(\hat{\rho}_{\mathrm{A}}^{(n-1)} \otimes \hat{\eta}_{\mathrm{b}}^{(\beta)}\right) \hat{U}^{\dagger}\right]\right)$. It is worth stressing that, at variance with the intrinsic bound where Eq. (18) can be seen as a consequence of Eq. (A1) via direct summation of the latter over all collisions, Eq. (A3) results in a weaker bound for the global entropy production of A than Eq. (19). Indeed by summing over the first $n$ collisions Eq. (A3) yields the inequality (20) with

$$
\Delta S_{\mathrm{B}}^{(n, \mathrm{loc})}:=\sum_{k=1}^{n} S\left(\hat{\rho}_{\mathrm{b}}^{(k)}\right)-n S\left(\hat{\eta}_{\mathrm{b}}^{(\beta)}\right),
$$

the bound being outperformed by (A2) due to entropy subadditivity, i.e., $\sum_{k=1}^{n} S\left(\hat{\rho}_{\mathrm{b}}^{(k)}\right) \geqslant S\left(\hat{\rho}_{B}^{(n)}\right)$, the $\hat{\rho}_{\mathrm{b}}^{(k)}$ being the reduced density matrix of the $k$ th ancillary system of $\hat{\rho}_{\mathrm{B}}^{(n)}$. As a matter of fact, Eq. (A4) is exactly the bound one would get from (A2) when removing all the intraparticle correlations between the subenvironment elements, i.e., by replacing $\hat{\rho}_{\mathrm{b}}^{(n)}$ with the product state formed by the reduced density matrices of its constituents $\hat{\rho}_{\mathrm{b}}^{(n)} \rightarrow \hat{\rho}_{\mathrm{b}_{2}}^{(n)} \otimes \hat{\rho}_{\mathrm{b}_{1}}^{(n)} \otimes \cdots \hat{\rho}_{\mathrm{b}_{n}}^{(n)}$. As explicitly noted in Ref. [31] this procedure will not affect the dynamics of A (and hence its entropy increase), yet at the level of the extrinsic lower bound gives worse performances than the one presented in Eq. (A2).

Finally we notice that a formal rewriting of the identity (12) for the CM reads as

$$
\beta \Delta Q_{\mathrm{A}}^{(n)}+\Delta S_{\mathrm{B}}^{(n)}=-S\left(\hat{\rho}_{\mathrm{B}}^{(n)} \| \hat{\rho}_{\mathrm{B}}^{(0)}\right),
$$

which implies $-\Delta S_{\mathrm{B}}^{(n)} \geqslant \beta \Delta Q_{\mathrm{A}}^{(n)}$ and which can be directly proven by direct evaluation of the various terms by enforcing the local energy conservation identity $\Delta Q_{\mathrm{A}}^{(n)}=-\Delta Q_{\mathrm{B}}^{(n)}$ discussed in the main text. A similar identity holds for the incremental entropy variations, i.e.,

$$
\beta d Q_{\mathrm{A}}^{(n)}+d S_{\mathrm{b}}^{(n)}=-S\left(\hat{\rho}_{\mathrm{b}}^{(n)} \| \hat{\eta}_{\mathrm{b}}^{(\beta)}\right)
$$

which upon summation over the collision index $n$ yields the inequality

$$
-\Delta S_{\mathrm{B}}^{(n, \mathrm{loc})} \geqslant \beta \Delta Q_{\mathrm{A}}^{(n)},
$$

anticipated in Eq. (21) of the main text.
[1] J. Goold, M. Huber, A. Riera, L. del Rio, and P. Skrzypczyk, J. Phys. A 49, 143001 (2016).

[2] C. Shannon, Bell Syst. Tech. J. 27, 379 (1948).

[3] C. Shannon, Bell Syst. Tech. J. 27, 623 (1948).

[4] H. Breuer and F. Petruccione, The Theory of Open Quantum Systems (Oxford University Press, Oxford, 2002).

[5] S. Alipour et al., Sci. Rep. 6, 35568 (2016).

[6] S. Vinjanampathy and J. Anders, Contemp. Phys. 57, 545 (2016).

[7] H. Weimer et al., Europhys. Lett. 83, 30008 (2008).

[8] R. Alicki, J. Phys. A: Math. Gen. 12, L103 (1979).

[9] P. Talkner, E. Lutz, and P. Hänggi, Phys. Rev. E 75, 050102 (2007).

[10] P. Ćwikliński, M. Studzński, M. Horodecki, and J. Oppenheim, Phys. Rev. Lett. 115, 210403 (2015).
[11] M. Lostaglio, D. Jennings, and T. Rudolph, Nat. Commun. 6, 6383 (2015).

[12] V. Cavina, A. Mari, and V. Giovannetti, Phys. Rev. Lett. 119, 050601 (2017).

[13] V. Scarani, M. Ziman, P. Štelmachovič, N. Gisin, and V. Bužek, Phys. Rev. Lett. 88, 097905 (2002).

[14] J. Rau, Phys. Rev. 129, 1880 (1963).

[15] M. Ziman and V. Bužek, Phys. Rev. A 72, 022110 (2005).

[16] T. A. Brun, Am. J. Phys. 70, 719 (2002).

[17] V. Giovannetti and G. M. Palma, Phys. Rev. Lett. 108, 040401 (2012).

[18] V. Giovannetti and G. M. Palma, J. Phys. B 45, 154003 (2012).

[19] S. Cusumano, A. Mari, and V. Giovannetti, Phys. Rev. A 95, 053838 (2017). 
[20] S. Lorenzo, A. Farace, F. Ciccarello, G. M. Palma, and V. Giovannetti, Phys. Rev. A 91, 022121 (2015).

[21] Z.-X. Man, Y.-J. Xia, and R. Lo Franco, Phys. Rev. A 97, 062104 (2018).

[22] J. Anders and V. Giovannetti, New J. Phys. 15, 033022 (2013).

[23] A. S. Holevo, Quantum Systems, Channels, Information: A Mathematical Introduction (de Gruyter Studies in Mathematical Physics, Berlin, Boston, 2012).

[24] D. Reeb and M. M. Wolf, New J. Phys. 16, 103011 (2014).

[25] H.-P. Breuer, J. Phys. B 45, 154001 (2012).

[26] V. Gorini, A. Kossakowski, and E. C. G. Sudarshan, J. Math. Phys. 17, 821 (1976).

[27] G. Lindblad, Commun. Math. Phys. 48, 119 (1976).
[28] P. Strasberg, G. Schaller, T. Brandes, and M. Esposito, Phys. Rev. X 7, 021003 (2017).

[29] H. Spohn, J. Math. Phys. 19, 1227 (1978).

[30] S. Deffner and E. Lutz, Phys. Rev. Lett. 107, 140404 (2011).

[31] S. Campbell, F. Ciccarello, G. M. Palma, and B. Vacchini, Phys. Rev. A 98, 012142 (2018).

[32] E. Baümer, M. Perarnau-Llobet, P. Kammerlander, and R. Renner, arXiv:1712.07128.

[33] S. Lorenzo, R. McCloskey, F. Ciccarello, M. Paternostro, and G. M. Palma, Phys. Rev. Lett. 115, 120403 (2015).

[34] M. Pezzutto, M. Paternostro, and Y. Omar, New J. Phys. 18, 123018 (2016).

[35] L. Diosi, T. Feldmann, and R. Kosloff, Int. J. Quantum Inform. 04, 99 (2006). 